\title{
Los paisajes sociales del piedemonte nororiental de la Sierra de Velasco, La Rioja (siglos III-IX d.C.)
}

\section{(1) Pablo Cahiza", Gabriela Sabatini"** y M. Lourdes Iniesta"}

\section{Resumen}

En este trabajo se abordan los patrones de asentamiento de las comunidades del piedemonte nororiental de la Sierra de Velasco (La Rioja, Argentina) en una perspectiva multiescalar y diacrónica entre los siglos III y IX d.C. Se utiliza una metodología regional de prospecciones de cobertura total y procesamiento y análisis de datos georreferenciados mediante un Sistema de Información Geográfica. El desarrollo combinado de análisis de distribuciones de frecuencia, cartografía vectorial, vecino próximo, regla tamañorango, buffers e interpolación kriging ofrecen diferentes acercamientos para la obtención de resultados espaciales que reflejan procesos socioeconómicos locales. El modelo de ocupación del espacio en el área se desarrolla desde estructuras de asentamientos independientes en las cuencas de Anjullón, Los Molinos y Anillaco, articulando paisajes pedemontanos que se van transformando desde el predominio y recurrencia de locaciones residenciales pequeñas hacia el 300-600 d.C., la concentración de población en locaciones de mayor complejidad y tamaño arquitectónico entre el 600 y 800 d.C. y la relocalización en partes altas del piedemonte y quebradas hacia el 800 d.C. Los espacios aldeanos generados se caracterizan por construir áreas integradas, compartidas por locaciones residenciales, espacios productivos y áreas de molienda.

\section{Social landscapes of Sierra de Velasco northeastern foothills, La Rioja $\left(3^{\text {rd }}-9^{\text {th }}\right.$ century A.D.)}

Recibido:

2 de mayo de 2017

Aceptado:

6 de febrero de 2018

\section{Palabras clave}

Paisajes aldeanos

Espacialidad

Sistema de Información

Geográfica

La Rioja

\section{Keywords}

Village landscapes

Spatiality

Geographic Information System La Rioja 
neighbor, size-rank rule, buffers and kriging interpolation offer different approaches to obtain spatial results that reflect local socio-economic processes. In turn, these articulate foothill landscapes are transformed, from the predominance and recurrence of small residential locations during A.D. 300-600; the concentration of population around more complex and architecturally larger sites between A.D. 600 and 800; and finally, the relocation to high foothills and canyons towards A.D. 800. The villages are characterized as integrated spaces that include residential areas, productive spaces and grinding locations.

\section{Introducción}

El desarrollo de diferentes acercamientos teórico-metodológicos sobre la construcción humana de paisajes sociales ha impulsado durante las últimas décadas un notable avance en el reconocimiento arqueológico de las relaciones espaciales hombrenaturaleza y hombre-hombre. Los avances tecnológicos en torno a la recolección de datos arqueológicos georreferenciados mediante el uso del geoposicionador satelital (GPS), potenciado por la posibilidad de análisis con software de Sistemas de Información Geográfica (SIG) y estadísticos, permitieron que los proyectos de investigación desarrollaran amplias bases de datos para el análisis de los cambios y continuidades de los procesos sociales en el tiempo.

Entendemos que la organización espacial de un paisaje es el resultado de diferentes procesos acumulativos de una serie de dinámicas impuestas en una determinada fracción temporal. Entonces, el análisis arqueológico de las espacialidades humanas permite un acercamiento que enriquece las posibilidades de explicar las interacciones del pasado dentro de las sociedades, entre ellas y con el medio ambiente (Anschuetz, Wilshusen y Scheick, 2001; Criado Boado, 1999, entre otros).

Nuestro interés se focaliza en la explicación de las dinámicas sociales de las comunidades que habitaron el piedemonte nororiental de la Sierra de Velasco en la provincia de La Rioja, Argentina, durante el primer milenio de la Era. El trabajo que presentamos aquí posee como punto de partida la resolución de un diseño de investigación arqueológico multiescalar (local, microregional y regional) enfocado en el estudio de la espacialidad humana y su relación con los diferentes componentes ecológicos del área y sus recursos.

El estudio de las actividades humanas y de la organización de las relaciones sociales fue abordado teniendo en cuenta como indicadores la frecuencia, el tipo y el tamaño de la infraestructura arquitectónica, tanto de la doméstica — residencial y productivacomo de la pública (sitios complejos con plataformas o montículos) y la distancia entre estos componentes. El diseño de investigación fue completado con los resultados de intervenciones de excavación, permitiéndonos desarrollar una perspectiva diacrónica y ajustar la temporalidad y materialidad de las dinámicas sociales. Finalmente proponemos un modelo de ocupación del espacio que tiene en cuenta procesos de crecimiento, concentración y abandono parcial del área.

\section{Antecedentes del problema de estudio}

El marco geográfico del proyecto abarca el piedemonte oriental del sector norte del cordón occidental de la Sierra de Velasco, en el departamento Castro Barros, provincia de La Rioja. El paisaje natural es semidesértico y posee un gradiente altitudinal caracterizado por un perfil de relieve de altos contrastes, con sectores montañosos de 
la Sierra de Velasco que alcanzan hasta un máximo de $4.000 \mathrm{~m}$ s.n.m., piedemontes surcados por pequeños arroyos de montaña (1.000 a 1.600 m s.n.m.) y la depresión en el fondo de valle (800 a $1.000 \mathrm{~m}$ s.n.m.). Esta se encuentra limitada por estribaciones del cordón oriental del Velasco - La Sierra de la Punta (2.000 m s.n.m.), en las estribaciones del cordón oriental del Velasco que sirve de límite a una cuenca aluvional que tiene al río de la Punta como colector estacional (Figura 1). La región del valle posee precipitaciones que alcanzan los $200 \mathrm{~mm}$ anuales y pertenece a la provincia fitogeográfica del monte; sin embargo, por su composición florística, las quebradas se asemejan más al Chaco serrano, con precipitaciones que pueden superar los $300 \mathrm{~mm}$ anuales. En tanto, las zonas altas de la sierra presentan sectores de pastizales especialmente atractivos para camélidos y ganado moderno.

En el piedemonte —entre los 1.000 y 1.200 m s.n.m - y asociados a los cauces de agua permanente, se emplazan los denominados pueblos de "La Costa" y sus pequeños oasis agrícolas: Santa Vera Cruz (el único a mayor altura: 1.500 m s.n.m.), San Pedro, Anjullón, Los Molinos, Anillaco, Aminga, Chuquis, Pinchas y Agua Blanca (Mamaní, 2008).

Hacia el inicio de la Era se producen los primeros paisajes agrícolas en el Noroeste argentino (NOA), generando lo que se ha denominado la "explosión" del 1er milenio de la era. Estos asentamientos de características aldeanas se desarrollan en distintos componentes ecológicos del NOA como puna, valles y quebradas y están integrados por espacios agrícolas, pudiendo haber asociación o separación espacial entre ellos (Lema, 2014).

Estas sociedades aldeanas construyeron paisajes sociales basados en la diferenciación arquitectónica de espacios de uso doméstico y otros de uso público en el que predominaba el ejercicio sobre los recursos simbólicos de la comunidad. Este modelo estaba anclado en tradiciones andinas y alcanzó una expresión ampliamente difundida con recursos arquitectónicos monumentales como montículos y plataformas. El complejo simbólico se completaba con los conjuntos artefactuales en los que adquirían una gran relevancia las representaciones felínicas y de personajes como el sacrificador (González, 1998; Gordillo, 2007; Scattolin, 2006, entre otros). En este contexto general se desarrollarán las comunidades del piedemonte nororiental del Velasco cuyo estudio presentamos aquí.

\section{Materiales y métodos}

La selección de las áreas de prospección se corresponde con las de las cuencas de los arroyos de Anjullón, Los Molinos y Anillaco en los sectores de quebrada y piedemonte de la Sierra de Velasco, entre las cotas de 1.300 y 1.800 m s.n.m. Los datos fueron relevados a partir de un diseño de prospección de cobertura total de tres unidades espaciales, dos de $20 \mathrm{~km}^{2}$ aproximadamente -Anjullón y Los Molinos-y la tercera de $40 \mathrm{~km}^{2}$ - Anillaco- Cada área de prospección fue subdividida en parches georreferenciados y asociados a límites naturales y culturales observables en el terreno. A su vez, se hicieron transectas uniendo los espacios que se encontraban entre las tres unidades, por lo que el área prospectada alcanzó una superficie de $112 \mathrm{~km}^{2}$.

Las categorías de sitios arqueológicos relevados fueron: locaciones residenciales, espacios agrícolas, morteros y combinaciones de presencia compartida de rasgos. Además se registraron concentraciones y lugares de hallazgo aislado (Lha) de materiales superficiales, especialmente cerámica.

Las locaciones que presentan construcciones del tipo residencial fueron categorizadas en: 


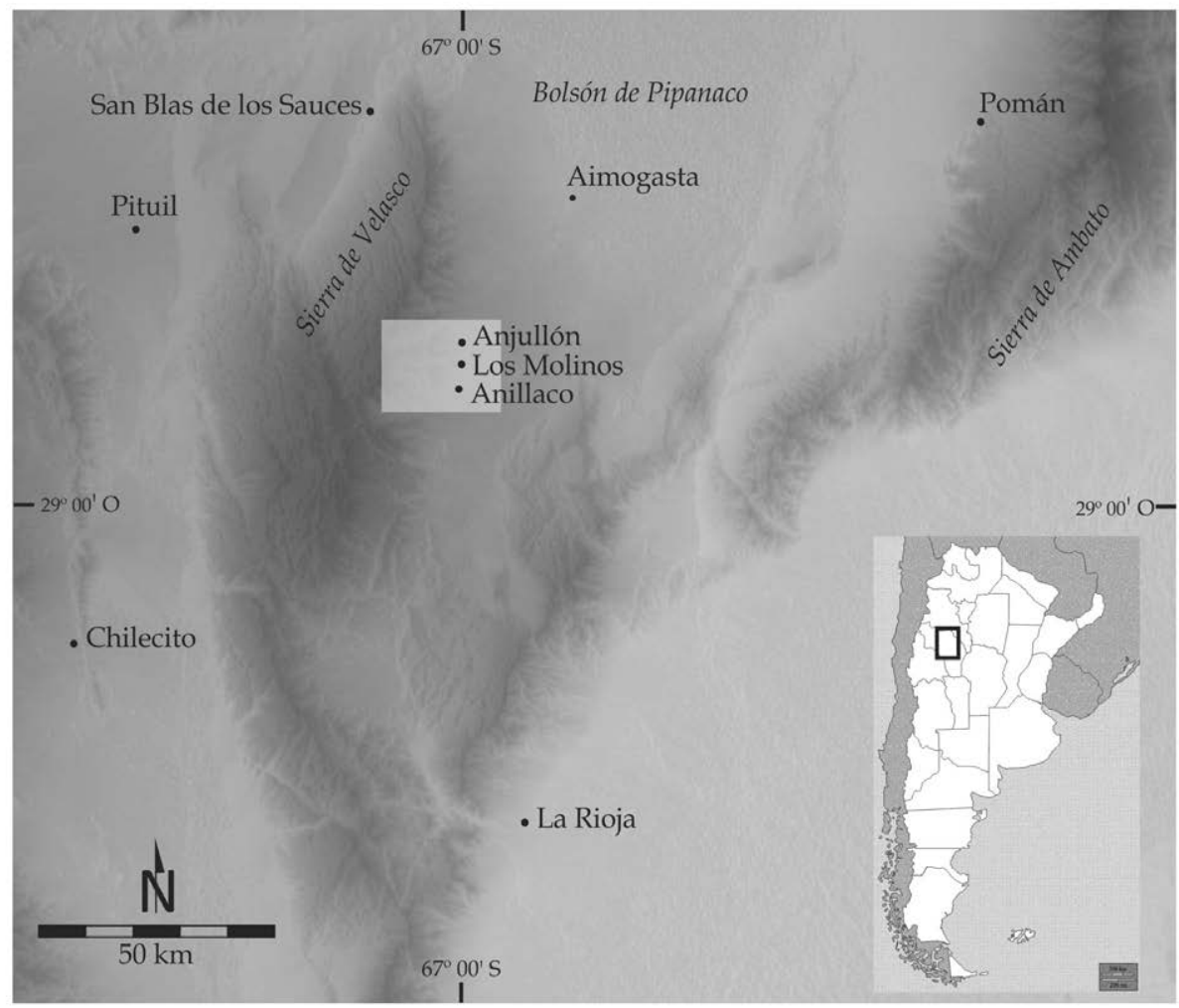

Figura 1. Mapa del área de estudio: Anjullón, Los Molinos y Anillaco (Castro Barros, La Rioja).

» Recintos simples: presentan uno o dos recintos de dimensión techable, de posible funcionalidad como habitación. En general presentan muros de piedra en hilera simple. En algunas ocasiones se presentan asociados a terrazas de cultivo. Los trabajos de excavación en Los Molinos involucraron la intervención de la locación residencial “Terraza 5", dos recintos pequeños asociados a campos aterrazados de cultivo (Cahiza, 2015). El componente cultural integrado por conjuntos cerámicos grises y naranjas con decoración incisa y cerámicas negras y grises pulidas - tradiciones Ciénaga y Allpatauca - fue datado en $1630 \pm 80$ años AP, 390-549 cal d.C. (LP - 3124, carbón vegetal).

» Recintos compuestos: presentan recintos clasificados como habitaciones asociados a uno o más espacios abiertos denominados patios. Uno de nosotros, Gabriela Sabatini, relevó y excavó en la cuenca de Anillaco el conjunto arquitectónico Uchuquita. La datación del piso de ocupación de Uchuquita 3 arrojó una antigüedad de $1760 \pm$ 50 años AP, 250-370 cal d.C. (LP-3259-carbón vegetal) (Sabatini y Garate, 2017).

» Recintos complejos: combinaciones de recintos habitacionales y patios que conforman "sectores". Pueden presentar otros espacios públicos, tales como plataformas y montículos, rampas y sectores de tránsito interno. Poseen muros de paredes de piedra en hilera doble. Esta categoría arquitectónica es la que más interés arqueológico ha recibido en la región. Se reproduce un mismo módulo constructivo que consiste en recintos pircados de hilada simple o doble de forma cuadrangular/rectangular y "patios" trapezoidales (Cahiza, 2015; Callegari et al., 2015; Mercado, 1993; Raviña y Callegari, 1991; Sabatini y Salminci, 2017). Raviña y Callegari (1991) realizaron investigaciones en la cuenca de Anillaco, allí efectuaron siete fechados radiocarbónicos en el sitio Faldeos de Anillaco I que oscilan entre los $1160 \pm 120$ y los $1440 \pm 120$ años AP (790 d.C. y 510 d.C., carbón vegetal), asociados a conjuntos cerámicos con decoraciones incisas en 
pastas naranjas y grises - tradiciones cerámicas Ciénaga y Allpatauca-y pintadas en negro y rojo sobre naranja con motivos felínicos de manchas de círculos concéntricos — tradición Aguada-. Gladys Mercado (1993) también realizó una excavación de un sitio residencial de esta categoría en la cuenca de Anjullón, el denominado por ella "Instalación 5", que fue datado en $1340 \pm 60$ años AP (carbón vegetal). Además, nuestros trabajos en la cuenca de Los Molinos se focalizaron en el sitio El Chañarcito, relevándolo y realizando excavaciones estratigráficas. Esos contextos fueron datados en $1330 \pm 70$ años AP, 659 a 841 cal d.C. (LP - 2959, carbón vegetal) y $1300 \pm 60$ años AP, 681 a 857 cal d.C. (LP - 2952, carbón vegetal) (Cahiza, 2015; Cahiza, Iniesta, Sabatini y Ots, en prensa). En Faldeos de Anillaco I y El Chañarcito, pudieron distinguirse elementos constructivos de probable funcionalidad pública: un montículo circular de 12 m de diámetro (Raviña y Callegari, 1991) y una plataforma de $64 \mathrm{~m}^{2}$ (Cahiza, 2015; Cahiza et al., en prensa; Cahiza, García, Llorca, Iniesta y Garate, 2017).

Emplazados en el ámbito ecológico del inicio de las quebradas del Velasco, se intervinieron dos sitios de difícil adscripción arquitectónica: "Quebrada de Anillaco" (Raviña y Callegari, 1991) y Loma de la Puerta (Cahiza, 2015) en las cuencas de Anillaco y Los Molinos. Ambos se encuentran construidos en posiciones defensivas sobre lomas con largas murallas de baja altura, con escasos recintos habitacionales o sin habitaciones en su interior, respectivamente. Las dataciones de sus pisos de ocupación son casi contemporáneas con los sitios descriptos en el párrafo anterior pero incorporan a su registro cerámico elementos de tradición Aguada tales como cerámicas naranjas pulidas con decoración en pintura negra y roja, secuencialmente más tardías que las anteriores.

Los espacios de producción agrícola están compuestos por campos aterrazados de superficies variadas que se caracterizan por presentar alineaciones de piedras separadas por intervalos de entre 2 y $4 \mathrm{~m}$ de distancia, dispuestas en una orientación perpendicular a la pendiente del terreno. En general se emplazan en pequeñas depresiones del piedemonte que parecen aprovechar áreas de captación hídrica de las precipitaciones y no se encuentran asociadas a infraestructura de acequias y canales. Los artefactos fijos de molienda fueron registrados en función de su localización, cantidad y diámetro de bocas de mortero.

Los datos fueron volcados en planillas de cálculo Excel y luego incorporados en un Sistema de Información Geográfica bajo una plataforma ArcGIS 10.1 generando cartografía analítica de los diversos componentes, especialmente residencial, productivo y de molienda. El procesamiento y análisis final fue realizado con software estadístico Past 3.02 (Hammer, Harper y Ryan, 2001) y Golden Surfer 8 (Golden Software Inc.). Con los datos relevados pudimos desarrollar una cartografía arqueológica digital en el que se representaron las distribuciones de sitios arqueológicos. A su vez también utilizamos cantidades y tamaños de atributos de los sitos (superficie, cantidades de recintos y bocas de morteros entre otros) para graficar escalarmente. Recurrimos a la estadística descriptiva para analizar diferencias en la distribución de sitios respecto a cada cuenca.

Uno de los atributos utilizados en nuestro análisis espacial fue la distancia entre las locaciones residenciales y la definición de un patrón distribucional. Nos aproximamos a ello a través del empleo del “vecino más próximo". Este test describe la distribución de una población en el espacio ya sea al azar o no y en este último caso mide el grado de regularidad o de agrupamiento de la distribución (Hodder y Orton, 1990). Los datos fueron procesados con el software estadístico Past 3.02 (Hammer et al., 2001) con la función Nearest neighbor.

Otra herramienta utilizada para el análisis de las distancias entre diferentes variables fue la aplicación del Multiple Ring Buffer de ARGIS 10.1 (ESRI). De esta manera pudimos 
convertir datos puntuales -áreas de cultivo- en múltiples polígonos circulares (Buffer) de 100 a $500 \mathrm{~m}$ de radio. Completamos este análisis incorporando un mapa vectorizado con la distribución de sectores residenciales con el fin de conocer el área de influencia y proximidad espacial entre estas categorías. Así, relacionamos diferentes distribuciones de locaciones específicas con posibles áreas de influencia basada en distancias.

Para analizar la jerarquía de espacios residenciales se realizó la estadística de la regla de tamaño-rango. Es un instrumento geográfico de análisis e interpretación que permite comparar la distribución jerárquica urbana de un sistema. En arqueología ha demostrado debilidades para las inferencias del modelo de curvas primo, primoconvexas y cóncavas como reflejos de sistemas equilibrados o desintegrados. Sin embargo posee fortalezas para describir la estructura de tamaños de los elementos residenciales de un sistema de asentamiento (especialmente cuando el registro está compuesto por arquitectura permanente), sobre todo cuando se toma como variable la superficie construida (Pearson, 1980).

Finalmente, con el objeto de mensurar la influencia de las locaciones residenciales y agrícolas realizamos análisis de interpolación. Esta herramienta estadística ofrece la ventaja de proyectar mapas o superficies continuas a partir de datos discretos (Drennan y Peterson, 2005), y en nuestro caso transforma los datos vectoriales de las superficies de las locaciones residenciales y terrazas de cultivo en datos raster con valores para cada celda o píxel del área de estudio. Existen varios tipos de interpolaciones espaciales, pero el método kriging es la mejor estimación de interpolación espacial, su mayor aplicabilidad se alcanza en casos con variables de mayor complejidad (Wheatley y Gillings, 2002). Utilizamos como valor $\mathrm{z}$ la superficie en $\mathrm{m}^{2} \mathrm{y}$ los datos fueron procesados en el software Golden Surfer 8 con la función grid/kriging proyectada en imágenes 3D.

\section{Resultados}

\section{Distribución general}

La muestra total está compuesta por 372 sitios arqueológicos, de los cuales 133 (35,75\%) son locaciones residenciales, 88 (23,66\%) espacios agrícolas, 107 (28,76\%) sitios de molienda, 37 (9,85\%) concentraciones y lugares de hallazgo aislado de cerámica superficie, y $7(1,88 \%)$ aleros y abrigos rocosos (Tabla 1$)$. Estas categorías de sitios fueron representadas en un mapa de distribución vectorial en el que se observa una clara asociación espacial entre las cuencas aluviales (cauces y conos) de los ríos de Anjullón, Los Molinos y Anillaco y las elecciones humanas de asentamiento, producción y molienda. Las áreas seleccionadas específicamente para residencia y explotación agrícola se encuentran mayoritariamente entre las cotas de 1.400 y 1.600 m s.n.m. (descripto por Raviña y Callegari, 1991 para la cuenca de Anillaco) (Figura 2).

Inicialmente consideramos a la muestra temporalmente indiferenciada, dada la escasa cantidad de dataciones absolutas, la tomamos como un conjunto discreto correspondiente a los siglos III al IX d.C. Más avanzado el texto intentaremos mejorar la resolución cronológica de algunos procesos reconocibles de la muestra, a partir de la interpretación de las tendencias de las dataciones radiocarbónicas locales.

Dentro de la distribución general de sitios por cuenca, se destaca la de Anjullón como la que posee mayor cantidad de sitios, con un total de 189 (50,81\% del total), seguida de Anillaco con 104 (27,96\%) y Los Molinos con 79 (21,23\%)

Sin embargo esta distribución adquiere otra forma cuando analizamos dimensiones distintas del registro y acudimos a la frecuencia de atributos diferentes de los sitios. Por 


\begin{tabular}{|l|c|c|c|c|c|c|c|c|c|c|}
\cline { 2 - 12 } & \multicolumn{2}{c|}{$\begin{array}{c}\text { Locaciones } \\
\text { residenciales }\end{array}$} & \multicolumn{2}{c|}{$\begin{array}{c}\text { Espacios } \\
\text { productivos }\end{array}$} & \multicolumn{2}{|c|}{ Áreas de molienda } & $\begin{array}{c}\text { Concentraciones } \\
\text { Lha }\end{array}$ & $\begin{array}{c}\text { Aleros y } \\
\text { abrigos }\end{array}$ & \multicolumn{2}{c|}{ Total } \\
\cline { 2 - 13 } & $\mathrm{n}$ & $\%$ & $\mathrm{n}$ & $\%$ & $\mathrm{n}$ & $\%$ & $\mathrm{n}$ & $\mathrm{n}$ & $\mathrm{n}$ & $\%$ \\
\hline Anjullón & 76 & 57,14 & 43 & 48,86 & 63 & 58,88 & 1 & 6 & 189 & 50,81 \\
Los Molinos & 21 & 15,79 & 19 & 21,6 & 23 & 21,49 & 16 & 0 & 79 & 21,24 \\
Anillaco & 36 & 27,07 & 26 & 29,54 & 21 & 19,63 & 20 & 1 & 104 & 27,96 \\
\hline Total & 133 & & 88 & & 107 & & 37 & 7 & 372 & \\
\hline
\end{tabular}

Tabla 1. Distribución de frecuencias relativas y absolutas por tipos de sitios.

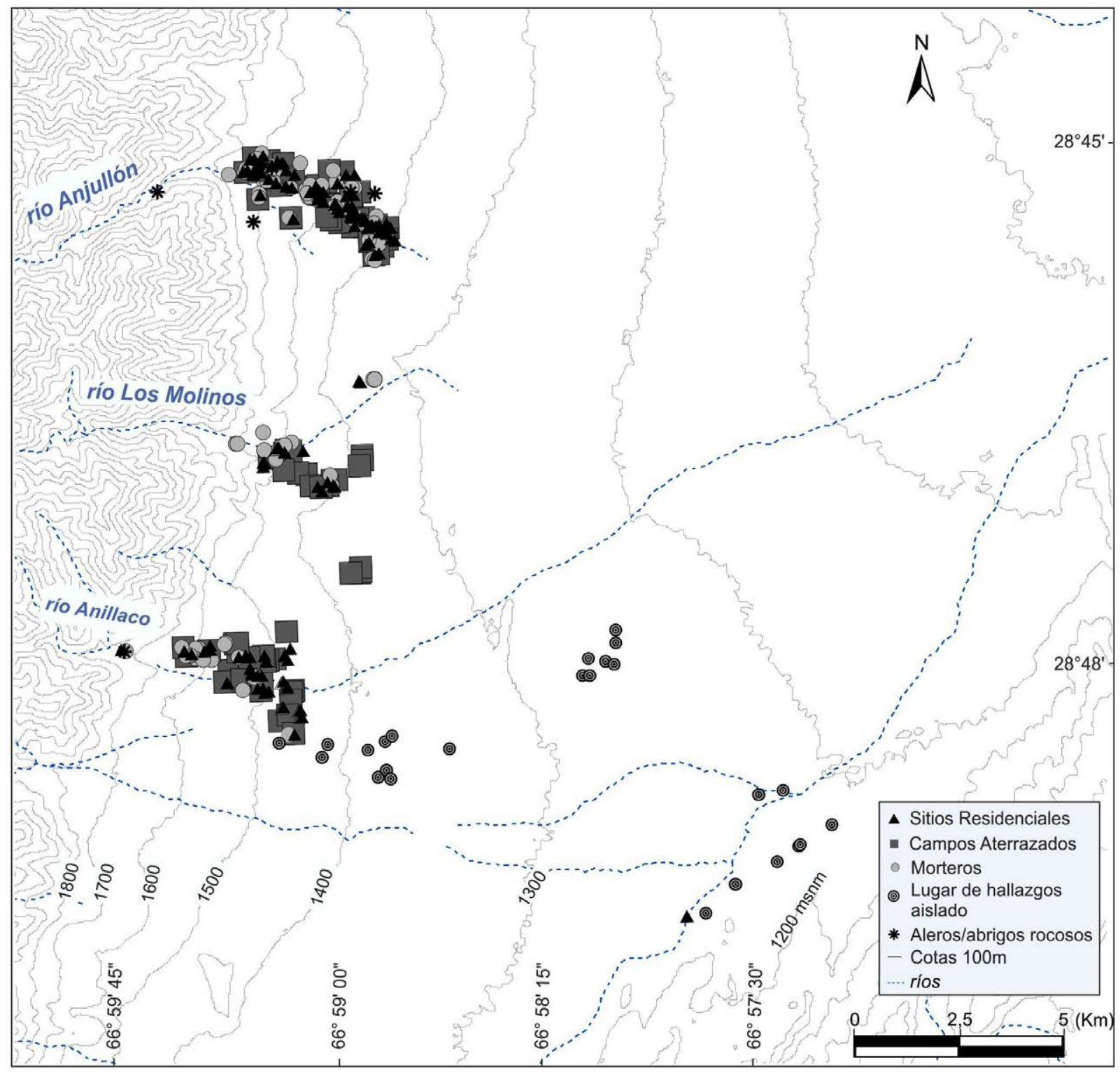

Figura 2. Mapa de distribución de sitios residenciales, productivos y de molienda en el área de prospección sistemática. 
ejemplo, si nos detenemos en las locaciones residenciales, Anjullón, la cuenca con más cantidad de registros de locaciones residenciales $(n=76,57 \%)$ posee la segunda área en $\mathrm{m}^{2}$ de superficie construida $\left(6.002 \mathrm{~m}^{2}\right)$, la media más baja de cantidad de recintos $(2,70$ recintos/locación) y de superficie media de las locaciones $\left(79 \mathrm{~m}^{2} /\right.$ locación). Anillaco, que concentra el $27 \%$ de las locaciones, es la cuenca con más superficie construida $\left(6.857 \mathrm{~m}^{2}\right)$ y una media ligeramente superior a la de Anjullón en cuanto a la cantidad de recintos por sitio $(3,4)$. Finalmente, Los Molinos es la cuenca con menos sitios en general y menos locaciones residenciales en particular con tan sólo 21 (15\%) y la menor superficie construida $\left(3.200 \mathrm{~m}^{2}\right)$, pero posee la media más alta de cantidad de recintos por locación $(6,90)$ y la superficie media también es la más elevada $\left(298 \mathrm{~m}^{2} /\right.$ locación $)$.

Es decir, en Anjullón se encuentra la mayor cantidad de locaciones, aunque predominan las de tamaño pequeño con pocos recintos y baja superficie construida, mientras que Anillaco posee la mayor cantidad de superficie construida y Los Molinos de menor cantidad de locaciones y superficie construida pero posee asentamientos de mayor tamaño que el de las otras cuencas.

El relevamiento general de espacios destinados a la producción agrícola es de 88 sitios que poseen una superficie total de 13,65 ha (Tabla 1). La distribución de estos espacios guarda algunas similitudes con el análisis de la distribución de las locaciones residenciales. Anjullón concentra el $49 \%$ de la muestra de terrazas de cultivo, sin embargo la superficie de cultivo relevada es de $46.489 \mathrm{~m}^{2}$, por detrás de Los Molinos que posee $50.314 \mathrm{~m}^{2} \mathrm{y}$ tiene el $22 \%$ de los sitios de terrazas de cultivo. Parte de la explicación del fenómeno puede deberse a las superficies medias de las parcelas agrícolas, mientras que en Anjullón el promedio es de $1.081,14 \mathrm{~m}^{2}$ en Los Molinos es de $2.648,11 \mathrm{~m}^{2}$. En tanto que en Anillaco hemos relevado la menor superficie de espacios de uso agrícola y el tamaño de sus parcelas (1.527,92 $\mathrm{m}^{2} /$ espacio productivo) se encuentra entre el promedio de Anjullón y Los Molinos, aunque es en esa cuenca donde se encuentra el espacio continuo de mayor tamaño dedicado a la agricultura con $27.825 \mathrm{~m}^{2}$ (Tabla 2).

Nuevamente resaltamos una tendencia similar a la del análisis de locaciones residenciales descripto anteriormente: la presencia de más sitios productivos y de menor tamaño en la cuenca de Anjullón respecto a la de Los Molinos, en la que menos espacios aterrazados poseen una mayor superficie de cultivo.

Si esta vez analizamos la muestra de sitios donde se identificaron instrumentos fijos de molienda encontramos un fenómeno similar al expuesto en los párrafos anteriores (Tabla 1). En este caso Anjullón posee la mayor cantidad de sitios ( $\mathrm{n}=63$ y 59\%) aunque las 111 bocas de mortero relevadas representan una media de 1,76 bocas/sitio de molienda, y el promedio resulta más bajo que el de Los Molinos $(\mathrm{n}=23$ y $21 \%$ de sitios y 54 bocas) de 2,35 bocas/sitio de molienda y el de Anillaco $(n=21$ y $20 \%$ de sitios y 58 bocas) que tiene la media más alta de 2,76 bocas/sitio de molienda.

El patrón de distribución de sitios muestra tres grandes concentraciones de sitios, una por cuenca (Figura 2), en el que aparecen entremezclados espacialmente locaciones residenciales, espacios productivos y áreas de molienda.

\section{Análisis de distancias}

Las distancias euclidianas entre las locaciones residenciales fueron obtenidas a partir de la aplicación del análisis del vecino más próximo en Past con datos proyectados en Universal Transversal Mercator (UTM) (Tabla 3). La muestra total tiene una distancia media de $118,47 \mathrm{~m}$ respecto al vecino más próximo con un nivel medio-bajo de agrupamiento y una $\mathrm{R}$ de 0,4029 . Si el análisis es realizado para la cuenca de Los Molinos, la distancia media calculada es de 149,91 m y el nivel de $r$ más alto, con 0,7522, 


\begin{tabular}{|c|c|c|c|c|c|c|c|}
\hline & \multicolumn{4}{|c|}{ Locaciones residenciales } & \multirow{2}{*}{\multicolumn{3}{|c|}{$\begin{array}{c}\text { Espacios Productivos } \\
\text { superficie }\left(\mathrm{m}^{2}\right)\end{array}$}} \\
\hline & \multicolumn{2}{|c|}{ recintos } & \multicolumn{2}{|c|}{ superficie $\left(m^{2}\right)$} & & & \\
\hline & $\mathbf{n}$ & media & área & media & área & media & rango \\
\hline Anjullón & 205 & 2,7 & 6.002 & 78,97 & 46.489 & $1.081,14$ & $10-5 \cdot 724$ \\
\hline Los Molinos & 76 & 6,91 & 3.253 & 298,72 & 50.314 & $2.648,11$ & $100-17.641$ \\
\hline Anillaco & 123 & 3,42 & 6.857 & 190,46 & 39.726 & $1.527,92$ & $8-27.825$ \\
\hline Total & 404 & 3,04 & 16.112 & 121,14 & 136.529 & $1.551,46$ & \\
\hline
\end{tabular}

Tabla 2. Frecuencia de atributos de locaciones residenciales y espacios productivos.

\begin{tabular}{|l|c|c|}
\cline { 2 - 3 } \multicolumn{1}{c|}{} & Distancia media $(\mathrm{m})$ & $\mathbf{R}$ \\
\hline Anjullón & 101,16 & 0,6557 \\
Los Molinos & 149,91 & 0,7522 \\
Anillaco & 98,68 & 0,6004 \\
\hline Total & $\mathbf{1 1 8 , 4 7}$ & $\mathbf{0 , 4 0 2 9}$ \\
\hline
\end{tabular}

Tabla 3. Análisis del vecino más próximo. Resultados de distancia media y $R$ de locaciones residenciales.

lo cual indica un patrón agrupado y mayor al de Anjullón, el cual presenta 101,16 m de distancia media y una $r$ de 0,6557 , en tanto que la distribución residencial de Anillaco es la de menor distancia media respecto a su vecino más próximo, 98,68 m, pero el $r$ menos agrupado con 0,6004 . Por lo tanto, la distribución de locaciones residenciales de toda la muestra presenta una agrupación media-baja, mientras que el análisis por cuenca refleja niveles de agrupamiento más altos, destacándose la distribución de Los Molinos.

La distribución de espacios residenciales y productivos posee un patrón de uso del espacio integrado, mixto, en el que no hemos identificado áreas de exclusividad residencial o agrícola. Este patrón es recurrente en las tres cuencas y puede ser reconocido en la Figura 3. Allí observamos el solapamiento entre la distribución vectorial de espacios residenciales y las áreas de influencia generada por buffer de hasta $500 \mathrm{~m}$ de radio. En general podemos establecer que una locación residencial se encuentra en un rango menor a los 100-200 m de un espacio agrícola. Esto refleja no solo la cercanía de sitios residenciales y sectores productivos sino también la ausencia de planificación supra-doméstica que signifique la asignación de espacios exclusivos para la residencia y práctica agrícola. A su vez, el área agrícola está emplazada en sectores de cursos de agua semipermanentes, hecho que, sumado a la ausencia de infraestructura hídrica, refuerza la idea de que las terrazas eran cultivadas a secano, es decir, regadas con el agua de las precipitaciones. El mapa da cuenta entonces que los sitios residenciales se encontraban dispersos entre las áreas agrícolas y, a su vez, estaban íntimamente relacionados con los cursos de agua - uno de los condicionantes de su ubicación - (Di Lillo, 2012). Esto lo evidencia la asociación espacial, aunque no implica ninguna relación de contemporaneidad ni de propiedad individual o colectiva de ése espacio productivo respecto de una locación residencial cercana.

\section{Jerarquización de locaciones residenciales}

La muestra de 133 locaciones residenciales fue subdividida según el criterio especificado anteriormente, delimitanto sitios integrados por recintos simples, compuestos y complejos 


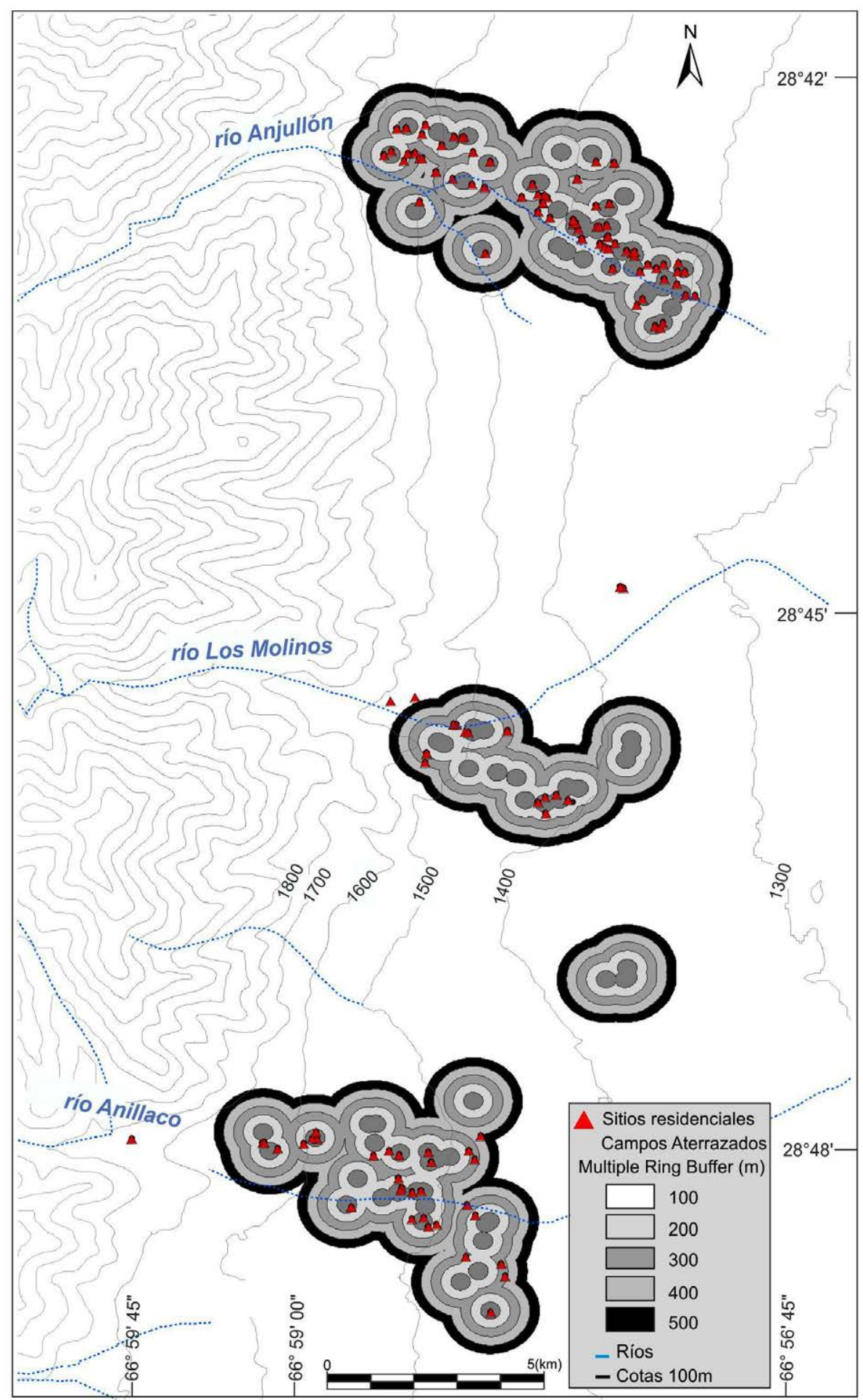

Figura 3. Mapa combinado de distribución vectorial de locaciones residenciales y buffers de espacios productivos.

(Tabla 4 y Figura 4). Las categorías representadas reflejan las conclusiones ya visualizadas en el análisis de las superficies medias de las locaciones residenciales (Tabla 2). Claramente se observa una mayor recurrencia de locaciones de recintos simples, especialmente en Anjullón. En el caso de las locaciones denominadas complejas, sólo cinco casos están presentes en cada cuenca y en un muy bajo número, pero además dos de ellas poseen 

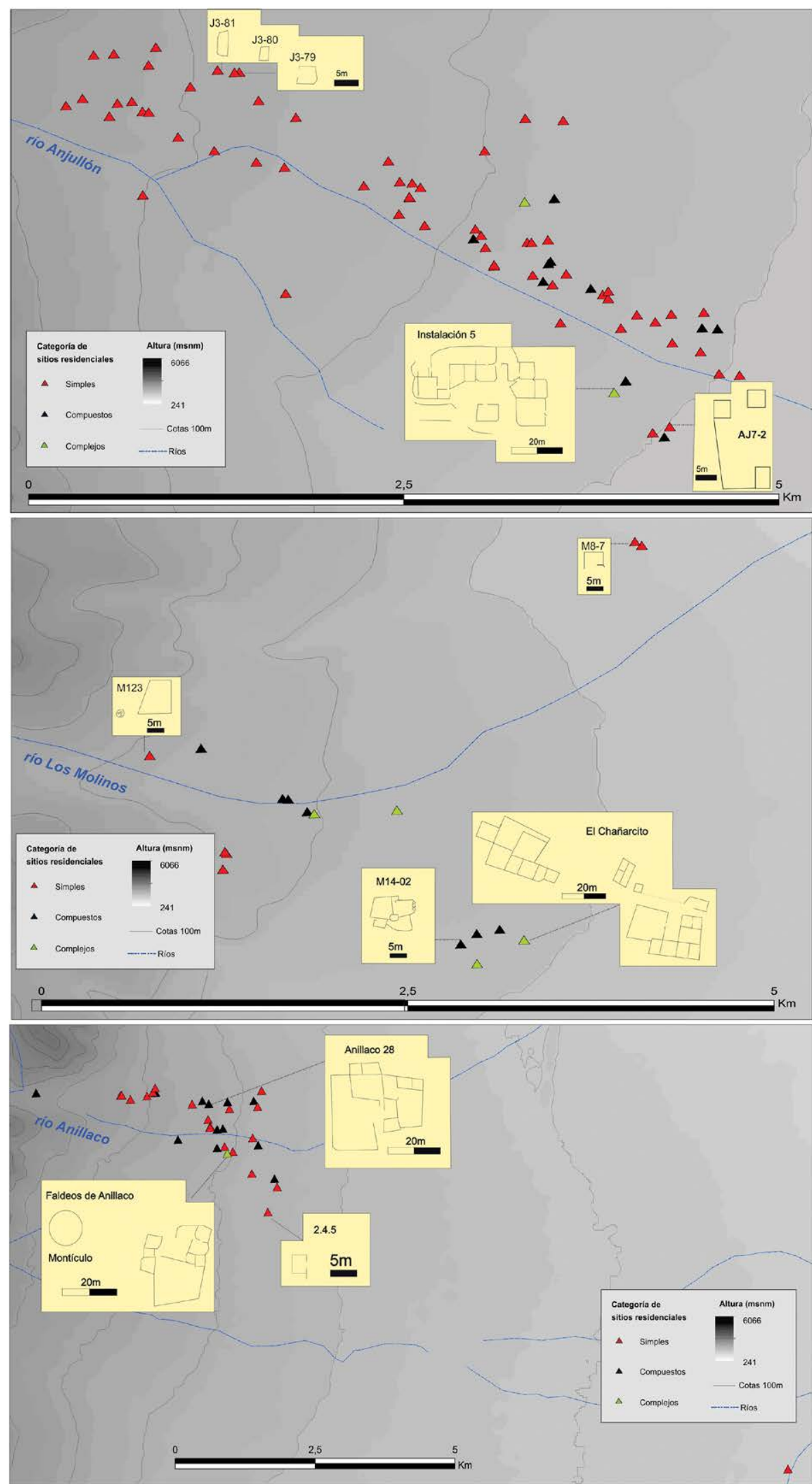

Figura 4. Mapa combinado de distribución vectorial de sitios por cuenca según categorías residenciales y ejemplos de plantas arquitectónicas. 


\begin{tabular}{|l|c|c|c|}
\cline { 2 - 4 } \multicolumn{1}{c|}{} & Simple & Compuesto & Complejo \\
\hline Anjullón & 59 & 15 & 2 \\
Los Molinos & 11 & 8 & 2 \\
Anillaco & 22 & 13 & 1 \\
\hline Total & 92 & 36 & 5 \\
\hline
\end{tabular}

Tabla 4. Distribución de sitios según categorías arquitectónicas.

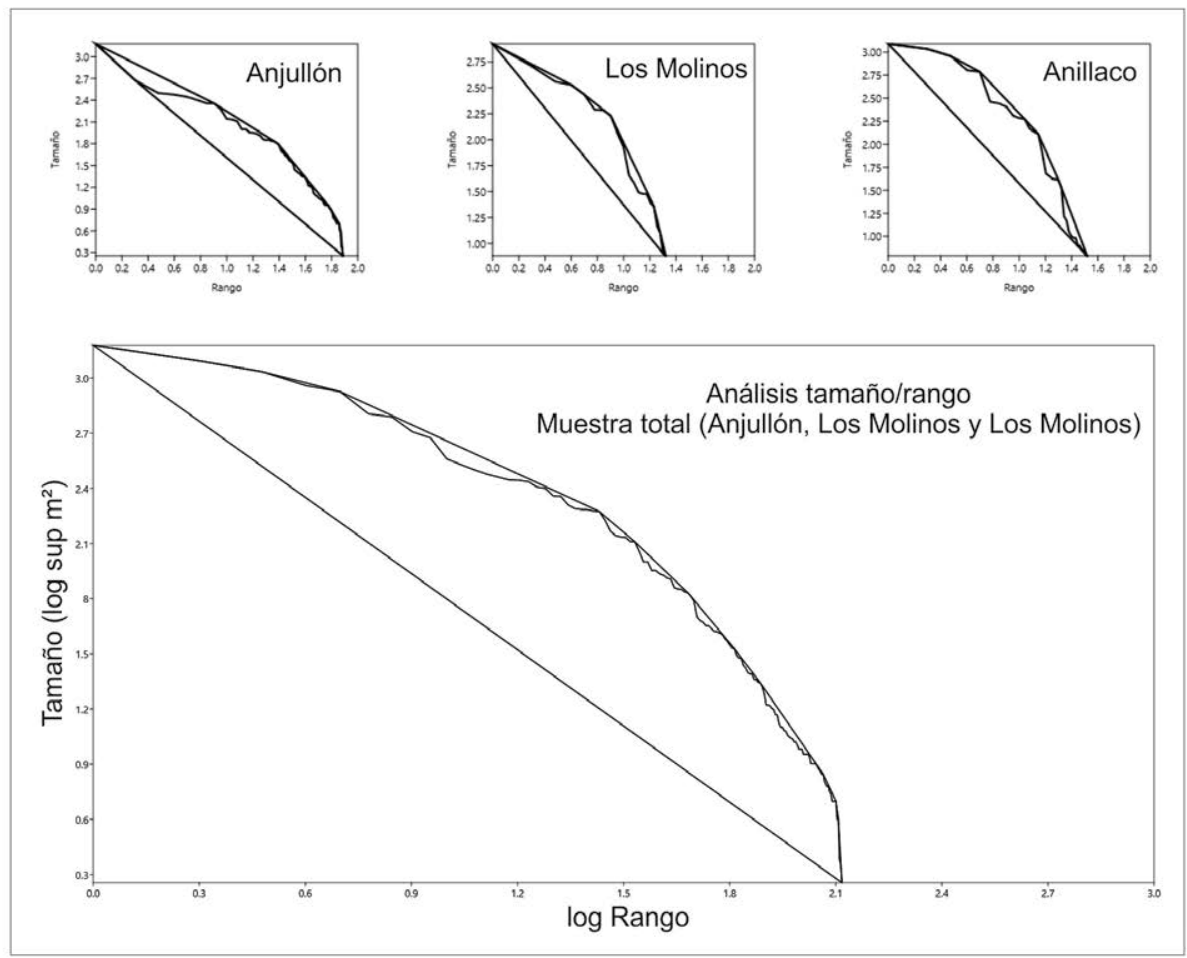

Figura 5. Análisis de la regla tamaño/rango de locaciones residenciales.

arquitectura pública - un montículo en Anillaco (Faldeos de Anillaco I; Raviña y Callegari, 1991) y una plataforma en Los Molinos (El Chañarcito; Cahiza, 2015)—.

La aplicación de la regla tamaño-rango nos brindó un acercamiento a la jerarquización de los asentamientos, nuevamente sobre la muestra total y sobre la de cada cuenca. La curva del área total nos muestra que no existe un asentamiento con una jerarquía regional que predomine sobre las tres cuencas. Los elementos que se encuentran en el primer puesto del rango general son los que se encuentran en el primer lugar de cada cuenca: Instalación 5, $1.510 \mathrm{~m}^{2}$ en Anjullón; El Chañarcito, $1.141 \mathrm{~m}^{2}$ en Los Molinos y Anillaco 28 (sensu Raviña y Callegari, 1991), $1.236 \mathrm{~m}^{2}$ en la muestra de Anillaco.

En la cuenca de Anjullón puede observarse en el principio de la curva un sistema equilibrado de escalaramiento entre los diversos órdenes de tamaños de los asentamientos, acentuándose en la segunda parte de la curva la influencia estadística de los asentamientos de los rangos más bajos, de tamaños pequeños. En el caso de Los Molinos y Anillaco, estas cuencas poseen sistemas menos equilibrados donde los sitios de segundo orden poseen una gran importancia, lo que influye decisivamente en la curva de la muestra total (Figura 5). 
Finalmente, elaboramos dos superficies suavizadas (Figura 6) resultado de la realización de dos interpolaciones kriging representadas en un modelo tridimensional. Una fue ejecutada con los datos vectoriales de superficie construida de las locaciones residenciales y la otra con la superficie de cultivo de los espacios productivos, obteniendo dos cartografías rasterizadas en 3D. Interpretamos los resultados como la representación de las influencias de las superficies construidas de los sitios de la categoría compleja y de los sitios de cultivo de mayor tamaño por cada cuenca.

En la cuenca de Anjullón se observa un pico correspondiente a Instalación 5 (Mercado, 1993), el sitio de la categoría compleja de mayor superficie y cantidad de recintos. También se destacan numerosos picos de baja altura correspondientes a una muestra de tamaños diversificada de bajo tamaño.

En cambio, las cuencas de Los Molinos y Anillaco muestran picos residenciales más suavizados y de relevancia areal más expandida en su base. El sitio El Chañarcito es el de influencia estadística más expandida de la cuenca en la interpolación residencial y la superficie de cultivo al norte del sitio el área productiva de mayor importancia.

En Anillaco los picos residenciales del sitio complejo Faldeos de Anillaco I y los sitios compuestos Anillaco 28 y Quebrada de Anillaco (Raviña y Callegari, 1991) son los que poseen más relevancia en la ponderación de sus superficies construidas. En la interpolación de espacios agrícolas, la influencia de "A2.10.terraza" (contiguo a Anillaco 28) es la más importante de la cuenca y del área de estudio, probablemente porque sea el espacio continuo de uso agrícola de mayor tamaño (2,7 ha).

\section{Discusión}

\section{Paisajes sociales y modelos de ocupación del espacio en el piedemonte oriental de la Sierra de Velasco}

Los espacios aldeanos son presentados en este trabajo desde la evidencia material, con énfasis en la residencia, agricultura y molienda, pero esto se relaciona únicamente con el tipo de registro espacial utilizado por nosotros para la aproximación al problema de estudio. Entendemos que estos paisajes son construcciones integradas por entramados sociales, económicos y simbólicos en el que se articulan las experiencias y trayectorias individuales y colectivas, además de las condiciones reales y las percibidas de la comunidad (De Feo, 2015; Korstanje, 2010)

Las elecciones para el emplazamiento de los lugares de residencia y de producción estuvieron fuertemente relacionadas con sectores elevados del terreno, de fácil acceso y cercanos a los cursos de agua de Anjullón, Los Molinos y Anillaco, especialmente entre los 1.400 y $1.600 \mathrm{~m}$ s.n.m. Esta elección podría ser el resultado de una conjunción de acciones conscientes con fines prácticos, en el que se destaca un componente de sustentabilidad ecológica (van der Leeuw, 2005) sobre la cual podrían haber operado otras decisiones grupales e individuales.

La configuración del espacio en la Sierra de Velasco refleja los patrones aldeanos de una sociedad que durante el primer milenio de la Era vinculó su vida doméstica con las tareas de producción agrícola, dando como resultado la construcción de un entramado compartido de espacios residenciales con unidades domésticas separadas entre sí pero a distancias cercanas. Esta asociación se observa claramente en la cartografía de buffers al representarse las locaciones residenciales entre las áreas productivas (Figura 3). Las estrategias económicas, sin embargo, no se limitarían exclusivamente a la producción 


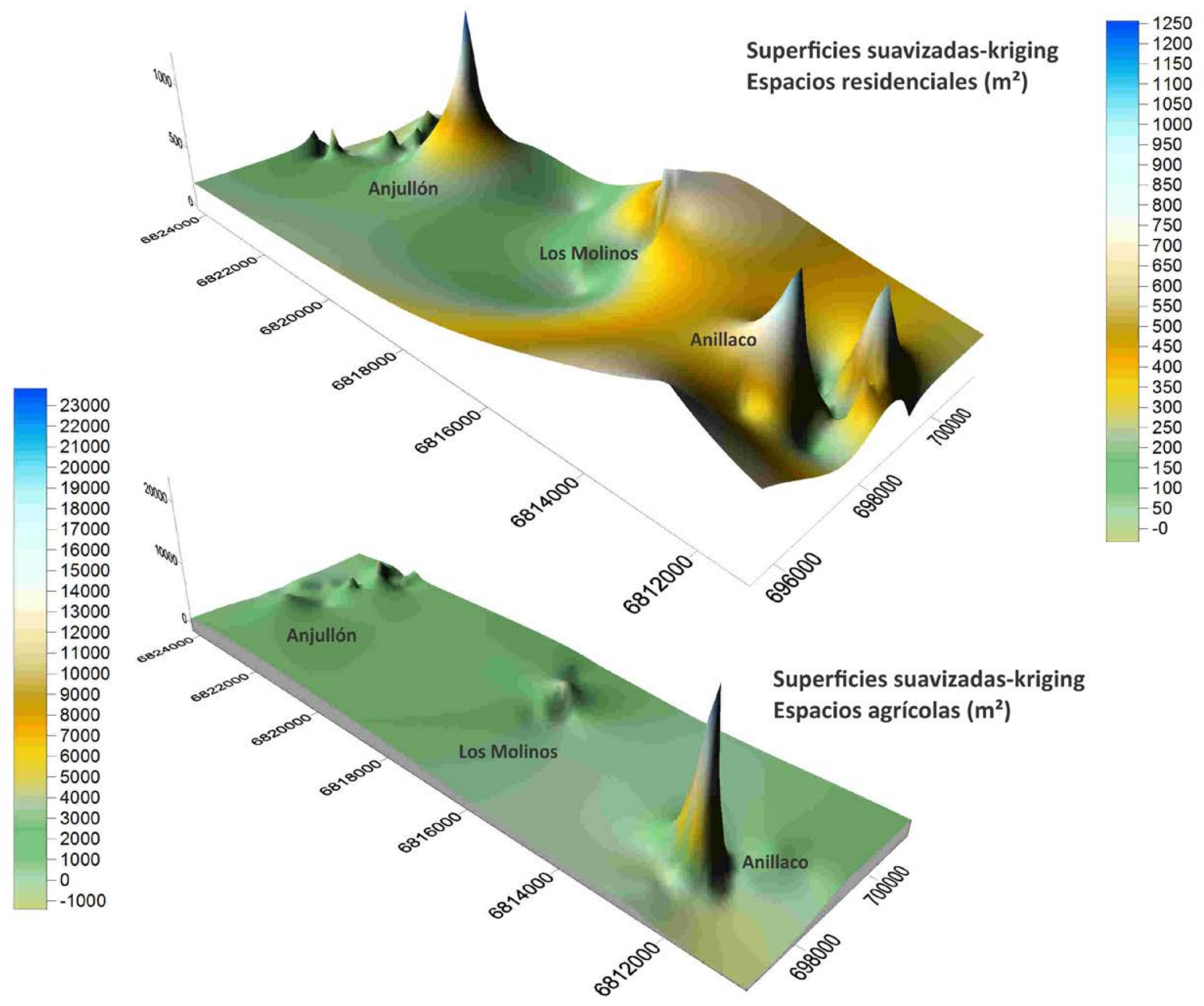

Figura 6. Análisis de interpolación kriging de locaciones residenciales (arriba) y espacios productivos (abajo) proyectados en coordenadas UTM.

agrícola sino que estarían complementadas con componentes extractivos como la recolección y la caza de camélidos (Cahiza et al., 2017).

La superficie total de campos aterrazados relevada es de aproximadamente 14 ha y el promedio por cuenca es de unas 4,5 ha, lo cual nos indica una intensidad baja de la producción agrícola. Esto surge de la comparación con sectores del NOA en los que la agricultura del primer milenio estuvo representada por espacios de cultivo de varios cientos de hectáreas (Quebrada de los Corrales en Tucumán; Valle de Ambato, Laguna Blanca y Tebenquiche Chico en Catamarca, entre otros) (Lema, 2014) y demuestran la importancia relativa o intensidad del uso de espacios de uso agrícola en el norte de la Sierra de Velasco. Relevamos una gran diversidad de tamaños de estos espacios, abundando los de menor superficie, que probablemente estuvieran más relacionados con una planificación de nivel familiar que con una comunitaria. Sin embargo, las excepciones a este patrón pueden estar constituidas por la presencia de los espacios aterrazados de mayor superficie en las proximidades de las locaciones residenciales de mayor envergadura (Figura 6).

Los análisis espaciales han definido un patrón descentralizado sin una estructura jerarquizada con influencia regional. Cada cuenca posee una ocupación del espacio 
autónoma en el que un sitio posee cierta predominancia sobre el resto. Esos sitios, que pueden ser identificados en Instalación 5 (Anjullón), El Chañarcito (Los Molinos), Anillaco 28 y Faldeos de Anillaco I (Anillaco), presentan la mayor superficie construida de sus respectivas cuencas, al tiempo que dos de ellos poseen arquitectura pública de montículo y plataforma y además están asociados a los espacios productivos de mayor superficie. Sin lugar a dudas estos espacios concentran la mayor interacción social teniendo en cuenta que la cercanía brinda mayores posibilidades de encuentros interpersonales y representan constructos sociales comunitarios (Drennan y Peterson, 2005; Kolb y Snead, 1997).

Por otra parte, las investigaciones que hemos desarrollado en El Chañarcito identificaron la presencia de un 75\% de conjuntos cerámicos de serie fina (sensu Bugliani, 2008), la existencia de áreas de consumo diferencial de camélidos y la jerarquización de espacios de prácticas de mayor visibilidad social como rasgos iniciales de desigualdad social (Cahiza et al., 2017). El modelado de superficies suavizadas por interpolación (Figura 6) presenta picos que representan de alguna manera la influencia de estos espacios, probablemente de alcance microregional.

El proceso de construcción de espacios simbólicos en Castro Barros representa desarrollos locales, pero puede ser relacionado con dinámicas similares en contextos macro-regionales de la provincia de La Rioja. Aunque están especialmente asociados al Formativo superior o período de Integración Regional (aproximadamente entre el 600 y el 900 d.C.) (Cahiza, 2015; Raviña y Callegari, 1991), en otros espacios del NOA se dan casos claramente anteriores. La construcción de un espacio público del tipo monticular en Anillaco, la plataforma en Los Molinos y un caso sobresaliente en La Cuestecilla en Pituil, Valle de Antinaco en la vertiente occidental del Velasco (Figura 1) (Callegari, Gonaldi, Spengler y Aciar, 2013; Callegari et al., 2015), constituyen ejemplos claros y simultáneos del incremento de la importancia de los espacios simbólicos en las sociedades del área. Adriana Callegari y colegas (2013) han asignado a este elemento arquitectónico una funcionalidad pública vinculada a sociedades Aguada, diferenciadas por su tamaño en "grandes" y "pequeñas", asignándole áreas de influencia "sub-regional" y "comunitaria” respectivamente. El caso del Chañarcito, al igual que Faldeos de Anillaco I, se encuentran en el rango de la categoría "pequeña”/ "comunitaria” de Callegari, con una superficie de $68 \mathrm{~m}^{2}$ y $113 \mathrm{~m}^{2}$ respectivamente, en correspondencia con la influencia estadística asignada por nosotros a los sitios complejos de cada cuenca.

La evidencia regional fue considerada inicialmente como si fuera parte de un bloque temporal único (siglos III al IX d.C.). Sin embargo, aunque la base de datos cronológica es aún exigua, nos permite resaltar algunas tendencias del proceso de ocupación del espacio en el área de estudio. En la Figura 7 realizamos un ordenamiento de las dataciones calibradas por radiocarbono de muestras de las tres microrregiones. Las tendencias representadas muestran dos claros agrupamientos: por una parte, las dataciones de Uchuquita 3 y Terraza 5 (en la parte inferior de la figura) podrían reunirse en un primer componente ocupacional, con un rango temporal que abarca desde el 300 al 600 d.C. aproximadamente. Por otro lado, Instalación 5, El Chañarcito y Faldeos de Anillaco I podrían integrar un segundo componente temporal con una cronología aproximada entre el 600 y 800 d.C.

Un tercer componente podría resultar de su definición por la combinación de fechados radiocarbónicos y tradiciones estilísticas cerámicas. Tanto Quebrada de Anillaco como Loma de La Puerta (Los Molinos) poseen dataciones que pueden incluirlos en el componente 2, sin embargo la presencia recurrente de conjuntos cerámicos más tardíos, de alfarerías de tradición Aguada (decoraciones geométricas y felínicas estilizadas en pintura negra y roja sobre superficies pulidas, círculos negros rellenos de cuadriculados en el mismo color y círculos negros rellenos en rojo) podrían justificar la presencia de 


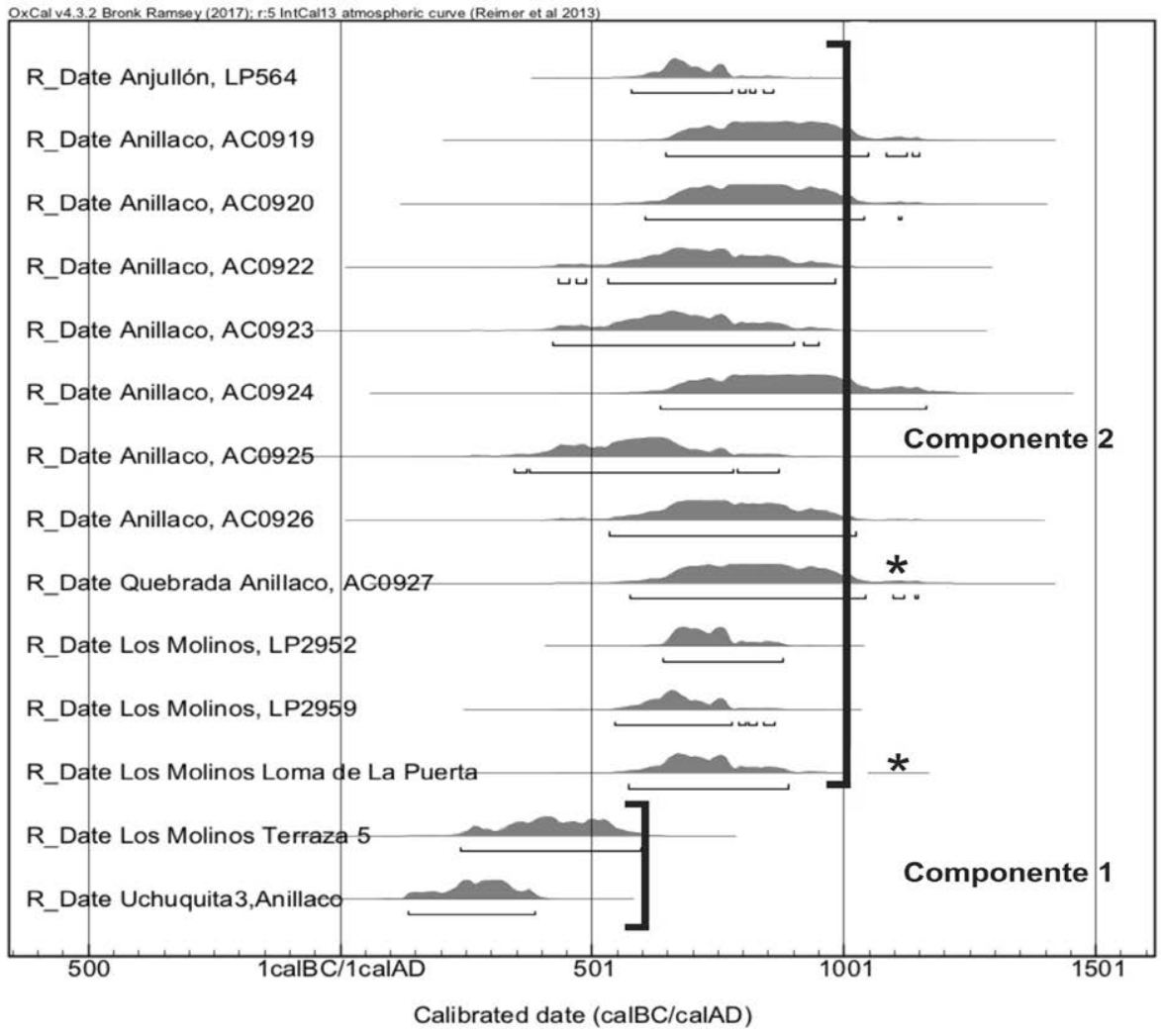

* Componente 3 (definido provisoriamente por contextos cerámicos)

Figura 7. Dataciones calibradas del piedemonte del Velasco.

un componente 3. Mientras los componentes 1 y 2 son representados por el uso del piedemonte entre las cotas de 1.400 y $1.600 \mathrm{~m}$ s.n.m., el tercero presenta locaciones emplazadas a mayor altura, en el inicio de las quebradas y en las quebradas mismas. Si seguimos este esquema temporal, entonces, proponemos la emergencia de grupos sedentarizados, de estructura descentralizada de base familiar, escasa desigualdad y asociaciones comunitarias hacia el 300 d.C. El proceso tendrá continuidad con el surgimiento de estructuras comunitarias aldeanas asociadas a arquitectura de uso público y a espacios de producción agrícola de mayor tamaño, proponiendo la presencia de fenómenos de intensificación productiva, integración residencial e interacción regional en torno al 600-800 d.C. extensible a todo el piedemonte oriental de la Sierra de Velasco. El incremento del tamaño de los espacios residenciales, la disminución en las distancias intra-sitios y el surgimiento de espacios de uso público sostendría la expectativa de crecimiento demográfico y el establecimiento de nuevas condiciones de interacción social reflejadas en un patrón de comunidades aldeanas. El abandono de la ocupación del piedemonte después del 800-1000 d.C. podría estar relacionado con el incremento de condiciones de aridización (Cahiza, 2015) que impulsó a los grupos locales a una reorganización del espacio social, lo que explicaría parcialmente el emplazamiento de las locaciones del componente 3 en tramos superiores de las cuencas ${ }^{1}$.

\section{Conclusiones}

La aplicación de un diseño de investigación de prospecciones microrregionales y su articulación con datos locales de excavación ha sido un esfuerzo metodológico positivo. 
La manipulación de los datos generados mediante software estadístico y Sistemas de Información Geográfica nos permitió identificar características generales de los paisajes sociales del piedemonte nororiental del Velasco y su evolución diacrónica.

Por un lado determinamos el aprovechamiento de suelos del piedemonte asociados a cursos de agua permanente (ríos Anjullón, Los Molinos y Anillaco), definimos la ausencia de un sistema centralizado regional, postulamos la autonomía comunitaria por cuenca, a nivel microrregional, integrada por un espacio aldeano combinado de locaciones residenciales y productivas y establecimos las características individuales de cada cuenca sobre la base de atributos de tamaño y tipo de locaciones residenciales y productivas.

Por otro lado, proponemos un modelo diacrónico de ocupación del espacio que señala un proceso heterogéneo de complejidad espacial creciente, desde capas de paisajes sociales con preponderancia de infraestructura de nivel familiar (300 al 600 d.C.); seguida del crecimiento de centros comunitarios en cada cuenca asociados a arquitectura pública ( $600 \mathrm{al} 800$ d.C.) y finalmente la selección de espacios protegidos en quebradas y el posterior abandono del sector (luego del 800 d.C.). En este sentido, los procesos sociales de construcción comunitaria parecen replicar mecánicas similares desarrolladas en otras partes del NOA durante el primer milenio d.C., aunque con temporalidades un poco más tardías en su inicio y adaptada a las condiciones ecológicas locales.

No obstante, este trabajo es un bosquejo de los paisajes sociales que presenta resultados con un grano grueso de resolución. Nuestras líneas de acción futuras incluyen la necesidad de incrementar la base de registro proveniente de intervenciones locales de excavación y la posibilidad de agregar perspectivas sobre las concepciones simbólicas de las comunidades y su reflejo en la construcción de paisajes.

\section{Agradecimientos}

A Jorge García Llorca, Enrique Garate, Agustina Acevedo, Silvina Rodríguez, Belén Lucero, Candela Fracchia, Lourdes Murri, Marina Cataldo, Franco García, Gonzalo García, Sebastián Puerto Mundt, Facundo Quintas, Franco Villegas, Andrés Rocha, Sebastián Rocher, Marcos Macchioli y Cristian Tivani que colaboraron en los trabajos de campo y laboratorio. Nuestras investigaciones han sido desarrolladas gracias al apoyo de un proyecto de la SECTyP de la Universidad Nacional de Cuyo y del soporte del Instituto de Ciencias Humanas, Sociales y Ambientales (INCIHUSA - CONICET Mendoza), del Centro Regional de Investigaciones Científicas y Tecnológicas de La Rioja (CRILAR), de la Facultad de Filosofía y Letras (Universidad Nacional de Cuyo), de la Secretaría de Cultura y la Dirección provincial de Patrimonio Cultural de la provincia de La Rioja, la Dirección de Cultura de la Municipalidad de Castro Barros y especialmente el cariño y hospitalidad de Adriana Rickert y Sebastián Fracchia, Soledad Gheggi, Pedro Salminci, María José Carvallo, Juan Narbona, Agustín Romero, Gerardo Vega y toda la comunidad de Los Molinos y Anillaco. A María Eugenia De Feo y Enrique Moreno, los organizadores del Simposio "Herramientas analíticas para el estudio del paisaje. Cruzando fronteras y tiempos" del XIX Congreso Nacional de Arqueología Argentina donde fue presentado este trabajo. 


\section{Dibliografía}

» Anschuetz, K. F., Wilshusen, R. H. y Scheick, C. L. (2001). An archaeology of landscapes: perspectives and directions. Journal of Archaeological Research, 9(2), 152-197.

"Bugliani, F. (2008). Consumo y representación en el sur de los Valles Calchaquíes (Noroeste argentino). Los conjuntos cerámicos de las aldeas del primer milenio A.D. Londres: BAR, International Series 1774.

"Cahiza, P. (2015). Un acercamiento espacial a los paisajes comunitarios formativos de Los Molinos -Castro Barros, La Rioja-. Relaciones de la Sociedad Argentina de Antropología, XL(1), 101-122.

»Cahiza, P., García, J., Llorca, M., Iniesta, L., y Garate, E. (2017). El Chañarcito: arquitectura, materialidad y consumo de un espacio residencial aldeano de la Sierra de Velasco, La Rioja (ca. 600 al 800 d.C.). Comechingonia, 21(1), 71-97.

" Cahiza, P., Iniesta, L., Sabatini, G. y Ots, M. J. (en prensa). Arquitectura y materialidad de la interacción social en la comunidad aldeana del Chañarcito, Los Molinos, La Rioja. Estudios Atacameños.

»Callegari, A., Gonaldi, M., Spengler, G. y Aciar, E. (2013). Construcción del Paisaje en el Valle de Antinaco, Departamento de Famatina, Provincia de La Rioja (o-130o AD). En A. Nielsen, I. Gordillo y J. M. Vaquer (Eds.), Tradición e Identidad (pp. 303-343). Quito: AbyaYala.

" Callegari, A., Gonaldi, M., Spengler, G., Rodríguez, M., Aciar, M., Pappalardo, R. y Wisnieski, M. (2015). Tras las huellas del Formativo. Norte de la provincia de La Rioja. En M. A. Korstanje, M. Lazzari, M. Basile, M. F. Bugliani, V. Lema, L. Pereyra Domingorena y M. Quesada (Eds.), Crónicas materiales precolombinas. Arqueología de los primeros poblados del Noroeste Argentino (pp. 247-275). Buenos Aires: Sociedad Argentina de Antropología.

»Criado Boado, F. (1999). Del Terreno al Espacio: Planteamientos y Perspectivas para la Arqueología del Paisaje. CAPA, 6, 1-82.

»De Feo, M. E. (2015). Puesta al día sobre el Formativo de la Quebrada del Toro (Salta, Argentina). En M. A. Korstanje, M. Lazzari, M. Basile, M. F. Bugliani, V. Lema, L. Pereyra Domingorena y M. Quesada (Eds.), Crónicas materiales precolombinas. Arqueología de los primeros poblados del Noroeste Argentino (pp. 277-311). Buenos Aires: Sociedad Argentina de Antropología.

》 Di Lillo, E. (2012). La casa y el campo en la quebrada de Los Corrales (El Infiernillo, Tucumán): Reflexiones sobre la espacialidad en el $1^{\circ}$ Milenio d.C. Comechingonia, 16, 85-104.

»Drennan, R. D. y Peterson, C. E. (2005). Early Chiefdom Communities Compared: The Settlement Pattern Record for Chifeng, the Alto Magdalena, and the Valley of Oaxaca. En R. E. Blanton (Ed.), Subsistence, Settlement, and Social Complexity (pp. 119-154). Los Ángeles: The Cotsen Institute of Archaeology Press.

» González, A. R. (1998). Arte precolombino. Cultura La Aguada. Arqueología y Diseños. Buenos Aires: Filmediciones Valero.

"Gordillo, I. (2007). Detrás de las paredes... Arquitectura y espacios domésticos en el área de la Rinconada (Ambato, Catamarca). En A. Nielsen, M. C. Rivolta, V. Seldes, M. Vázquez y P. Mercolli (Eds.), Producción y circulación prehispánicas de bienes en el sur andino (pp. 65-98). Córdoba: Brujas. 
» Hammer, Ø., Harper, D. A. T. y Ryan, P. D. (2001). PAST: Paleontological Statistics software package for education and data analysis. Palaeontologia Electronica, 4(1), http:// palaeo-electronica.org/2001_1/past/issue1_01.htm (Acceso: 15 de abril, 2017).

» Hodder, I. y Orton, C. (1990). Análisis Espacial en Arqueología. Barcelona: Crítica.

» Kolb, M. J. y Snead, J. (1997). “It's a small world after all”: comparative analyses of community organization in archaeology. American Antiquity, 62(4), 609-628.

» Kornstanje, M. A. (2010). Producción y consumo agrícola en el Valle del Bolsón (19922005). En M. A. Kornstanje y M. Quesada (Eds.), Arqueología de la Agricultura: Casos de Estudio en la Región Andina Argentina (pp. 48-75). San Miguel de Tucumán: Ediciones Magna.

»Lema, V.S. (2014). Boceto para un esquema: domesticación y agricultura temprana en el Noroeste argentino. Revista Española de Antropología Americana, 44(2), 465-494.

» Mamaní, M. (2008). Sierra de Velasco. Producto y limitantes de su potencial hídrico. La Rioja: Edular.

» Mercado, G. (1993). El yacimiento arqueológico de Anjullón. Investigación Preliminar. Anales de Arqueología y Etnología, 48/49, 91-103.

» Ortiz de Malmierca, M. (2001). “Loma Pircada” Estudios arqueológicos en los faldeos del Velasco. Chuquis departamento Castro Barros La Rioja (Argentina). Serie Informes de investigación, 2, 1-38.

»Pearson, Ch. E. (1980). Rank-Size Distributions and the Analysis of Prehistoric Settlement Systems. Journal of Anthropological Research, 36(4), 453-462.

» Raviña, G y Callegari, A. (1991). La Presencia Aguada en el departamento de Castro Barro (La Rioja). Palimpsesto. Revista de Arqueología, 1, 50-70.

»Sabatini, G. y Garate, E. (2017). Espacialidad y materialidad de las unidades domésticas tempranas -ca. 300-60o D.C.- de Anillaco (La Rioja, Argentina). Comechingonia, 21(14), 99-122.

» Sabatini, G y Salminci, P. (2017). Los paisajes aldeanos de la cuenca del río Anillaco, Sierra de Velasco, La Rioja (ca. 300-80o dC.). Revista del Museo de Antropología, 10 (Suplemento especial 1), 7-12.

»Scattolin, M. C. (2006). De las comunidades aldeanas a los curacazgos en el noroeste argentino. Boletín de Arqueología PUCP, 10, 357-398.

» van der Leeuw, S. (2005). Climate, Hydrology, land use, and environmental degradation in the lower Rhone Valley during the Roman period C.R. Geoscience, 337, 9-27.

»Wheatley, D. y Gillings, M. (2002). Spatial Technology and Archaeology. The archaeological application of GIS. Nueva York: Taylor \& Francis. 